\title{
Neural Correlates of Finger Gnosis
}

\author{
Elena Rusconi, ${ }^{1,2,3}$ Luigi Tamè, ${ }^{4}$ Michele Furlan, ${ }^{5}$ Patrick Haggard, ${ }^{2}$ Gianpaolo Demarchi, ${ }^{4}$ Michela Adriani, ${ }^{4}$ \\ Paolo Ferrari, ${ }^{4}$ Christoph Braun, ${ }^{4,6,7,8}$ and Jens Schwarzbach ${ }^{4,8}$ \\ ${ }^{1}$ Department of Neurosciences, University of Parma, I-43100 Parma, Italy, ${ }^{2}$ Institute of Cognitive Neuroscience, University College London, London WC1N \\ 3AR, United Kingdom, ${ }^{3}$ Division of Psychology, Abertay University, Dundee DD1 1HG, United Kingdom, ${ }^{4}$ Centre for Mind/Brain Sciences, University of \\ Trento, I-38123 Mattarello, Italy, ${ }^{5}$ Department of Psychology, Royal Holloway, University of London, Egham TW20 0EX, United Kingdom, ${ }^{6}$ MEG-Center, \\ ${ }^{7}$ Werner Reichardt Centre for Integrative Neuroscience, Eberhard-Karls-University of Tübingen, D-72076 Tübingen, Germany, and ${ }^{8}$ Department of \\ Psychology and Cognitive Science, University of Trento, I-38068 Rovereto, Italy
}

Neuropsychological studies have described patients with a selective impairment of finger identification in association with posterior parietal lesions. However, evidence of the role of these areas in finger gnosis from studies of the healthy human brain is still scarce. Here we used functional magnetic resonance imaging to identify the brain network engaged in a novel finger gnosis task, the intermanual in-between task (IIBT), in healthy participants. Several brain regions exhibited a stronger blood oxygenation level-dependent (BOLD) response in IIBT than in a control task that did not explicitly rely on finger gnosis but used identical stimuli and motor responses as the IIBT. The IIBT involved stronger signal in the left inferior parietal lobule (IPL), bilateral precuneus (PCN), bilateral premotor cortex, and left inferior frontal gyrus. In all regions, stimulation of nonhomologous fingers of the two hands elicited higher BOLD signal than stimulation of homologous fingers. Only in the left anteromedial IPL (a-mIPL) and left PCN did signal strength decrease parametrically from nonhomology, through partial homology, to total homology with stimulation delivered synchronously to the two hands. With asynchronous stimulation, the signal was stronger in the left a-mIPL than in any other region, possibly indicating retention of taskrelevant information. We suggest that the left PCN may contribute a supporting visuospatial representation via its functional connection to the right PCN. The a-mIPL may instead provide the core substrate of an explicit bilateral body structure representation for the fingers that when disrupted can produce the typical symptoms of finger agnosia.

Key words: body structure representation, finger gnosis, intraparietal circuits

\section{Introduction}

Knowledge about the arrangement of body parts is a fundamental form of self-awareness. However, there is little systematic research on how body structure representations (BSRs) are formed and what brain substrates support them.

BSRs are assumed to organize body parts and their spatial relations in a posture-independent layout (Serino and Haggard, 2010). The strongest evidence for their existence is autotopoagnosia after middle-temporal or parietal lesions of the dominant hemisphere (Schwoebel and Coslett, 2005). Autotopagnosic pa-

Received July 23, 2013; revised April 30, 2014; accepted May 7, 2014.

Author contributions: E.R., P.H., C.B., and J.S. designed research; E.R., L.T., G.D., M.A., and P.F. performed research; C.B. and J.S. contributed unpublished reagents/analytic tools; E.R., L.T., and M.F. analyzed data; E.R., L.T., M.F., P.H., C.B., and J.S. wrote the paper.

This work was supported by grants from the European Commission (MERG-CT-2007-046511) and Ministero dell'Istruzione, dell'Università e della Ricerca (RBFR08EHZT) to E.R., L.T. was supported by a Marie Curie Individual Fellowship (COFUND) from the European Commission and Provincia Autonoma di Trento (40101908). C.B. was supported by the Werner Reichardt Centre for Integrative Neuroscience of the University of Tübingen. P.H. was supported by EU FP7 Project Virtual Embodiment and Robotic Re-Embodiment. We thank Chris Chambers, Andreas Kleinschmidt, the editors, and two anonymous reviewers for their insightful comments on a previous version of the manuscript.

C.B. and J.S. contributed equally to this work.

The authors declare no competing financial interests.

Correspondence should be addressed to Dr. Elena Rusconi, Department of Neurosciences, University of Parma, Via Volturno 39/E, I-43100 Parma, Italy. E-mail: elena.rusconi@gmail.com.

DOI:10.1523/JNEUROSCI.3119-13.2014

Copyright $\odot 2014$ the authors $\quad 0270-6474 / 14 / 339012-12 \$ 15.00 / 0$ tients can move and point to body parts when touched but fail to point to them on verbal instruction or on a diagram (Poeck and Orgass, 1971; Semenza and Goodglass, 1985). Because fingers are more similar to one another than other body parts, finger agnosia was initially described as a light version of autotopagnosia (Gerstmann, 1940). However, it may be absent in autotopoagnosic patients (De Renzi and Scotti, 1970). Kinsbourne and Warrington (1962) redefined finger agnosia as a bilateral impairment in appreciating the position of individual fingers among others on tactile stimulation without sight. Neurological (Gainotti et al., 1972) and stimulation studies (Rusconi et al., 2005) identified bilateral parietal regions as the substrates for finger gnosis. While the right hemisphere may contribute to visuospatial functions implied by typical finger gnosis tasks, left posterior parietal lobes may support a core representation of finger gnosis (Rusconi et al., 2005).

Although the correlates of BSRs in the healthy human brain have been investigated (Corradi-Dell'Acqua et al., 2009), similar evidence on BSRs for the fingers is still scarce. We recently developed a modified version of Kinsbourne and Warrington's (1962) classic in-between test, the intermanual in-between task (IIBT), for use with healthy participants (Rusconi et al., 2009a; Fig. 1). In the IIBT, participants decide whether the finger distance between two touches on one hand is the same as the finger distance between two touches on the other hand. The IIBT is a direct finger 


\section{In-Between Task}

(Kinsbourne and Warrington, 1962)
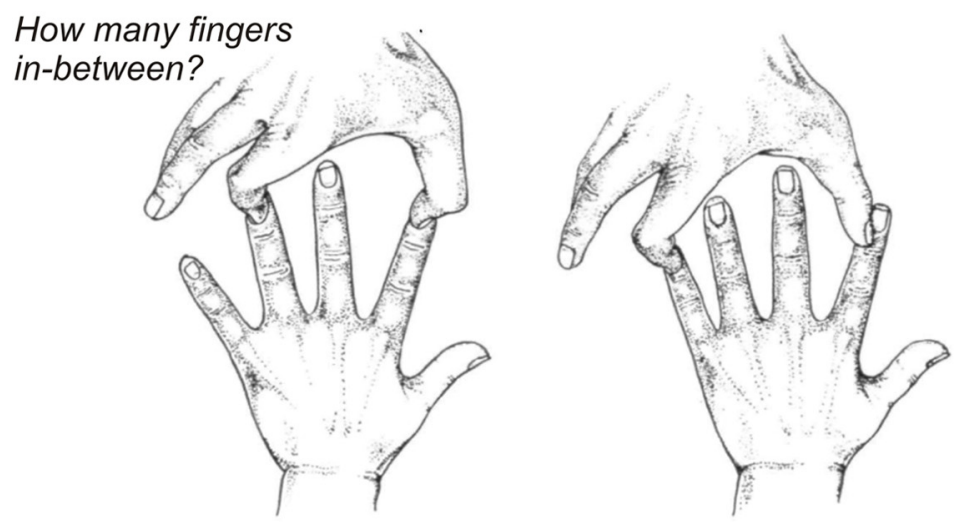

Intermanual In-Between Task

(Rusconi et al., 2009a)

Same or different?

\begin{tabular}{|c|c|c|c|c|c|c|c|c|}
\hline \multirow[b]{2}{*}{$\begin{array}{c}\text { Stimulation } \\
0\end{array}$} & \multicolumn{2}{|c|}{$n$} & & \multicolumn{2}{|c|}{$v$} & \multicolumn{2}{|c|}{5} \\
\hline & & & & & & & & \\
\hline Response & \multicolumn{2}{|c|}{ "same" } & \multicolumn{2}{|c|}{ "same" } & \multicolumn{2}{|c|}{ "different" } & \multicolumn{2}{|c|}{ "different" } \\
\hline $\begin{array}{l}\text { Fingers in } \\
\text { common }\end{array}$ & \multicolumn{2}{|c|}{2} & \multicolumn{2}{|c|}{0} & \multicolumn{2}{|c|}{0} & \multicolumn{2}{|c|}{1} \\
\hline \multirow{2}{*}{$\begin{array}{l}\text { Number of } \\
\text { fingers in } \\
\text { between }\end{array}$} & LH & RH & LH & RH & LH & RH & LH & RH \\
\hline & 0 & 0 & 1 & 1 & 1 & 2 & 2 & 0 \\
\hline
\end{tabular}

Figure 1. Top, In-between task. Two fingers are touched simultaneously on the same hand and the subject is to state the number of fingers in-between the ones touched in each trial. Adapted from Kinsbourne and Warrington (1962), with permission. Bottom, IIBT. Green dots on fingers indicate sites of stimulation. $\boldsymbol{A}$, No fingers in-between on either hand: a "same" answer is required. Full homology is present. Therefore, this pattern is classified as a Total Homology trial. $\boldsymbol{B}, 0$ ne finger in-between on either hand: a "same" answer is required. No homology is present, therefore this pattern is classified as a No Homology trial. C, One finger in-between on the left hand, two fingers in-between on the right hand: a "different" answer is required. $\boldsymbol{D}$, Two fingers in-between on the left hand, no fingers in-between on the right hand: a "different" answer is required. Only partial homology is present, since the index finger of both hands is stimulated, therefore this pattern is classified as a Partial Homology trial. Adapted from Rusconi et al. (2009a), with permission. LH = Left Hand; RH = Right Hand.

gnosis task but also elicits an implicit signature effect of finger identity processing, the homology effect. When the same fingers are touched on both hands (i.e., Total Homology condition), low-level sensory representations suffice to select the correct response (Iwamura et al., 2001; Tamè et al., 2011, 2012). Because primary sensory codes are short-lasting (Allison et al., 1992; Mauguière et al., 1997; Wühle et al., 2011), the homology shortcut is available when both hands receive tactile stimulation simultaneously but not with intermanual stimulation delay, where sustained use of finger gnosis is necessary (Rusconi et al., 2009a). In the present study, we measured brain activity while participants performed the IIBT and a matched control task in separate blocks. Homology and intermanual stimulation delay varied at the trial level. We expected a network of regions for finger gnosis to emerge from the IIBT > control task contrast. Moreover, parametric decrease in brain activity with increasing homology in synchronous stimulation trials and high engagement in asynchronous stimulation trials would identify core correlates of finger gnosis in the healthy human brain.

\section{Materials and Methods}

Participants. Thirteen participants (mean age, 27 years; SD, 4 years; 6 females) took part in the functional magnetic resonance imaging (fMRI) study. All reported normal or corrected to normal vision and somatosensation. They reported no history of psychiatric or neurological disorders, and no current use of any psychoactive medication. All participants were right-handed except for one who was lefthanded. An additional independent sample of 10 participants (mean age, 31 years; SD 7 years; 5 females; all right-handed) was also recruited for a brief behavioral follow-up experiment. Written informed consent was obtained before participation. The study was performed in accordance with the 1964 Declaration of Helsinki (Seoul, 2008) and was approved by the ethics committee of the University of Trento.

Stimuli and apparatus. Tactile stimuli were provided by 10 four-channel piezoelectric stimulators (Quaerosys, www.quaerosys.de) attached to each of the 10 fingertips with Velcro tape to ensure constant contact force between the fingers and the stimulation devices throughout the testing (Fig. 2A). Stimulator transducer voltage controlled displacements of a piezo-crystal and enabled protrusion or retraction of a plastic pin (diameter, $1 \mathrm{~mm}$; maximal skin indentation, $1.2 \mathrm{~mm}$ ). The devices gave a $1 \mathrm{~s}$ buzz-like vibration $(20 \mathrm{~Hz})$ to the stimulated fingertips. Wave signal intensity could be varied between trials and hands (although it was always the same for the two fingers within a hand during the behavioral training and the fMRI session) and could take two different levels ( $60 \%$ of maximum stimulator output and the intensity at individual threshold level previously determined outside the scanner, then also verified in a dry run before the experiment) for both the experimental and the sensory control task. To avoid distortions caused by the stimulation equipment in the MR environment, a ferrite low-pass filter was applied to the signals entering the MR room.

Foot responses were requested during the experiment. The mapping between foot (left, right) and response alternative (same, different) was balanced within conditions and pseudorandomly reassigned in every trial, with the constraint that no more than three consecutive trials had the same assignment (Fig. $2 B, C$ ). Visual stimuli for response-to-foot mapping were delivered using a liquidcrystal projector (Epson EMP 7900; refresh rate, $60 \mathrm{~Hz}$; resolution, $1280 \times 1024$ pixels) and were visible to the participants through a mirror positioned above the head coil.

Procedure. Before the fMRI experiment, participants underwent two behavioral testing sessions, one aimed to establish individual somatosensory thresholds at the fingers and one aimed to provide training with the experimental and control tasks.

Pre-experiment. We first obtained a measure of individual sensitivity to intermanual intensity differences. To this end, stimulators were attached to participants' left and right index fingers. We then asked participants to pay attention to the intensity of tactile stimulation under their fingertips 

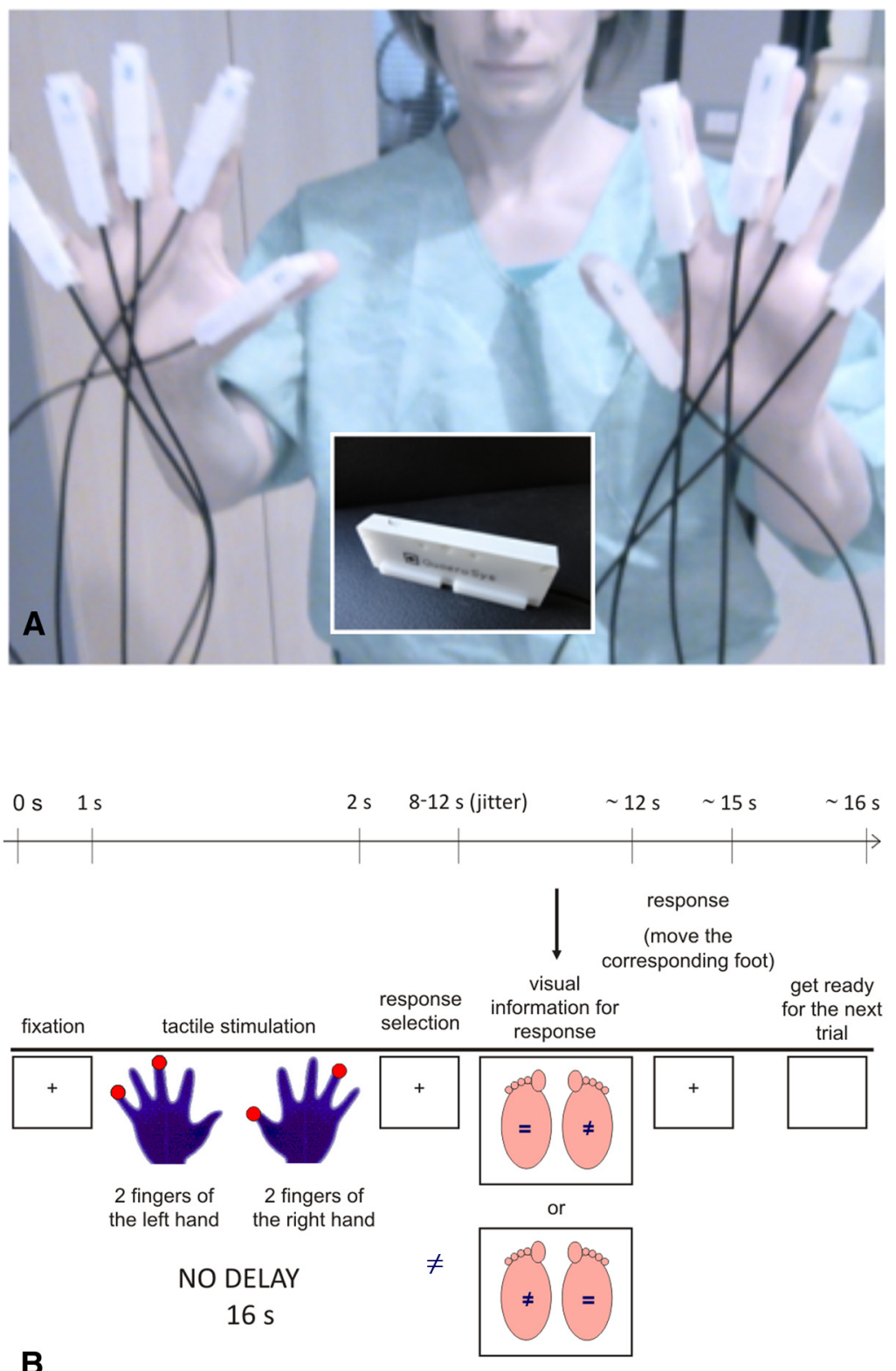

B

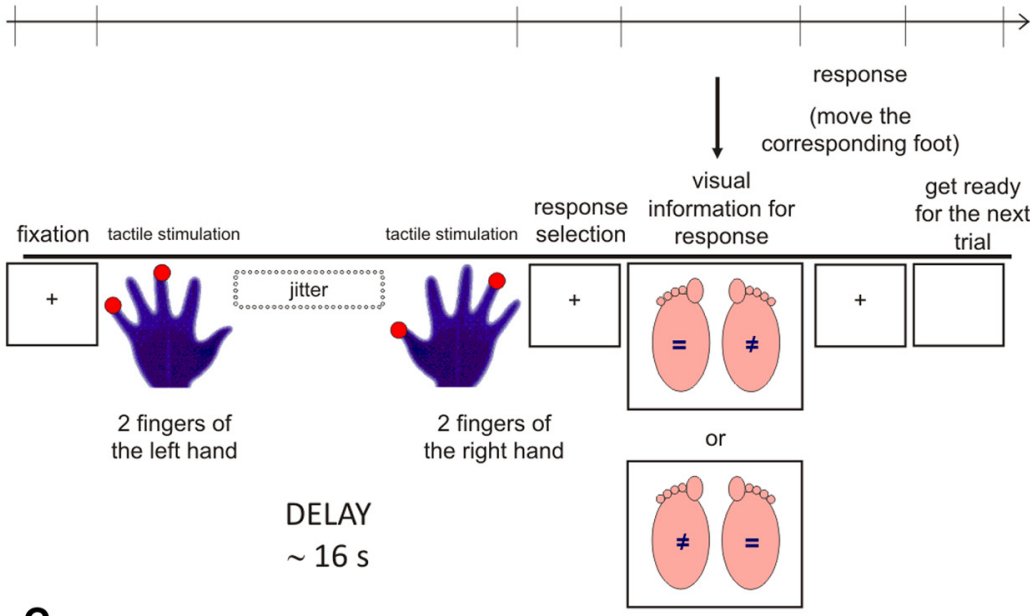

while wearing insulating headphones. Insulating headphones were used to mask any sounds from the tactile stimulators, because they could be used to infer differences in intensity. On each trial, two stimuli lasting $1 \mathrm{~s}$ each were delivered in rapid succession (interstimulus interval, $500 \mathrm{~ms}$ ), one on the left index finger and one on the right index finger, in random order. Participants said "yes" when they perceived any intensity difference between hands and "no" when stimulation intensity seemed identical. To obtain individual thresholds, we used a two-alternative forced-choice staircase procedure, with fixed step sizes of $10 \%$ in the upward direction and $5 \%$ in the downward direction.

This procedure was shown to be adequate, in combination with a two-down/one-up rule, to converge to our target performance level of $80 \%$ correct (García-Pérez, 1998). After either 20 reversals or 100 trials had elapsed, an individual value was calculated for the higher-intensity stimulus (e.g., $94 \%$ of maximum stimulator output). We kept the lowerintensity level fixed at $60 \%$ of maximum stimulator output throughout the procedure. Because our threshold identification procedure focused on the discrimination between the intensity of two stimuli rather than on simple detection of a tactile stimulus, we chose our "lower-intensity" stimulus well above detection threshold. We then used $60 \%$ maximum stimulator output and the individual $80 \%$ discrimination threshold intensity as starting values in the following pilot

$\leftarrow$

Figure 2. A, Piezoelectric stimulators are shown as they were attached to participants' fingertips. A stimulating pin (inset) was placed under the center of each fingertip. $\boldsymbol{B}$, Schematic description of the time course of an experimental trial in the No Delay condition. C, Schematic description of the time course of an experimental trial in the Delay condition. Each trial started with the presentation of a white central fixation cross on a dark background (colors are inverted in the figure for printing), which remained on the screen during stimulation. After a variable time jittered between 5 and $7 \mathrm{~s}$, four tactile stimuli were delivered to the participant's fingers, each of them lasting $1000 \mathrm{~ms}$ ( 2 simultaneous stimuli on the left hand: S1; 2 stimuli on the right: $\$ 2)$. In the No Delay condition, S1 and S2 were delivered at the same time, whereas in the Delay condition they were separated by an interstimulus interval (ISI) jittered between 3000 and $5000 \mathrm{~ms}$. S2 was followed by a waiting interval jittered between 3000 and $5000 \mathrm{~ms}$, after which visual information on the response-foot assignment was shown for $2000 \mathrm{~ms}$, followed by a black screen with a white central fixation cross for an additional $1000 \mathrm{~ms}$. Participants were instructed to select their answer in the time available between the end of a stimulus and the appearance of a pseudorandom response-to-foot assignment display. They were expected to move the appropriate foot based on their previously selected response (i.e., same or different; same or different refer to distance between stimulations on the two hands in the IIBT, intensity of stimulation on the two hands in the IINT). Disappearance of the black display with a central fixation cross announced an imminent new trial. Each trial lasted on average $16 \mathrm{~s}$. 
session for low-intensity and high-intensity stimuli respectively.

To familiarize participants with the IIBT and the sensory control task, $\geq 2$ series of 15 trials each were performed for both tasks before entering the scanner. Tactile stimuli were delivered to the thumb, index, middle, ring, and small fingertips of either hand using the $10 \mathrm{MR}$-compatible stimulators described above. In each trial, participants received two stimulations on different fingers of each hand. Stimulation could be delivered synchronously or with a delay between hands (Fig. $2 B, C$ ). In the IIBT, we asked participants to judge whether the finger distance between stimulations was the same or different between hands. In the sensory control task (Rusconi et al., 2009a), we asked participants to judge whether stimulation intensity was the same or different between hands, regardless of identity of the stimulated fingers. Finger distance and intensity were orthogonally manipulated and identical stimulation patterns were used in the IIBT and the control intermanual-intensity task (IINT; Rusconi et al., 2009a, their Experiment 3), both in the training and in the following fMRI session. In the training phase, we encouraged participants to ask for any clarifications they may need and gave them the opportunity to exercise until they felt confident with the tasks.

fMRI experiment. During fMRI, participants rested their unseen hands palm down in a comfortable posture, one on each side of their abdomen. We used the same vibrotactile stimulators as in the pre-experiment session and instructed participants not to touch their hands together to avoid creating a loop with their arms, which might induce electrical currents in peripheral nerves.

The fMRI session comprised two tasks that had been practiced outside the scanner: the IIBT and IINT. In the IIBT, participants judged whether distance between the two stimulated fingers was the same or different across the two hands, regardless of stimulation intensity. In the IINT, participants judged whether fingers received stimulation of equal or different intensity between hands, regardless of stimulation location and finger distance. The two stimulated fingers within each hand always received stimuli with the same intensity but stimulation could be more intense on one hand than on the other (i.e., intensity could differ only between hands). We adjusted intensity levels to individual sensitivity, using as starting values $60 \%$ of maximum stimulator output as lower intensity and the individual threshold level to obtain a discrimination accuracy of $80 \%$ as higher intensity. Stimulation was provided with randomly varying levels of homology (i.e., number of common fingers stimulated), between 0 and 2 fingers in common. We refer to the condition in which the same fingers were stimulated on both hands as Total Homology. The condition in which different fingers were stimulated on both hands is referred to as No Homology. Partial Homology is the term used when a finger was stimulated on both left and right hands, but the other fingers differed between hands (Fig. 1). We programmed visual and vibrotactile stimulations using the ASF software (Schwarzbach, 2011), based on Matlab (Mathworks) Psychotoolbox-3 (Brainard, 1997) for Windows. Lateralized foot responses, indicating the responses "same" or "different," were video-recorded from inside the scanner room with an MR-compatible videocamera as a backup, while the experimenter registered responses outside the scanner room by pressing two buttons on a response box (Lumina LP-400 system, Cedrus) with his dominant hand, in correspondence with the participants' feet movements. In case of hesitations or corrections, the trial was rechecked off-line and the most appropriate response was reported. If the two feet were moved simultaneously, the trial was logged as error. In case of two subsequent responses, we considered the first. The proportion of such instances over the total number of trials was negligible, likely because participants were given an ad hoc time window to select their response before actually implementing it. We used closed-ear headphones (Serene Sound, Resonance Technology) to reduce the noise caused by the operation of the scanner and any noise leaking from the tactile stimulator (see Fig. $2 B, C$ ).

Behavioral follow-up. Participants performed the IINT under standard (i.e., practice-like: silent room; seated posture; normal temperature, $\approx 26^{\circ} \mathrm{C}$ ) and then under fMRI-like environmental conditions. In the fMRI-like session, they performed the IINT in a cooled room $\left(\approx 18^{\circ} \mathrm{C}\right)$, while lying supine and hearing via headphones the recorded noise of a scanning sequence (echo-planar imaging).
Design. In the fMRI study, we used two levels of delay (No Delay, Delay), three levels of homology (No Homology, Partial Homology, Total Homology), and two tasks (IIBT, IINT) in an event-related design. Each participant took part in one scanning session comprising eight successive runs. In each run, 30 trials were presented in a pseudorandom order, so that delay and homology conditions, response (same, different), and response foot (right, left) were evenly distributed across runs. At the beginning of each run, and before the onset of the acquisition sequence, participants were informed about which task they were expected to perform throughout the entire run. They performed IIBT in four of the eight runs and IINT in the remaining four, for a total of 120 trials per task and 240 trials per session (Rusconi et al., 2009a, their Experiment 3). We counterbalanced the order of tasks between participants according to two series, both starting and ending with the same task (order 1: IIBT-IINT-IINT-IIBT-IIBT-IINT-IINT-IIBT; order 2: IINTIIBT-IIBT-IINT-IINT-IIBT-IIBT-IINT). Each run lasted on average $621 \mathrm{~s}$. Before the first and after the last trial of a run, a blank screen was presented for 16 and $20 \mathrm{~s}$, respectively.

In the behavioral follow-up, participants performed a first half-run of the IINT ( 60 randomized trials) in practice-like condition and then a full run of the IINT (120 randomized trials) in fMRI-like conditions. Delay and Homology were manipulated in both sessions, to extract (within participants) the same behavioral markers that were described by Rusconi et al. (2009a) under standard laboratory conditions and in the current fMRI study under scanning conditions.

Data acquisition. MR scans were acquired using a Bruker Biospin MedSpec 4 T MR scanner and an eight-channel birdcage head coil. Functional images were acquired using $\mathrm{T} 2^{*}$-weighted gradient-recalled echo-planar imaging. An additional scan was performed to measure the point-spread function of the acquired sequence, which served for the correction of distortions from high-field imaging (Zeng and Constable, 2002). Twenty-five contiguous slices were acquired in interleaved ascending order, which provided almost full-brain coverage, with a TR (repetition time) of $1500 \mathrm{~ms}$ [voxel resolution, $3 \times 3 \times 3 \mathrm{~mm}$; TE, $33 \mathrm{~ms}$; flip angle (FA), $75^{\circ}$; field of view (FOV), $192 \times 192 \mathrm{~mm}$; gap size, $0.45 \mathrm{~mm}$ ]. A T1-weighted anatomical scan of each participant was also acquired at higher resolution [MP-RAGE; $1 \times 1 \times 1 \mathrm{~mm}$; FOV, $256 \times 224 \mathrm{~mm} ; 176$ slices; GRAPPA (generalized autocalibrating partially parallel acquisition) with an acceleration factor of 2; TR, $2700 \mathrm{~ms}$; TE, $4.18 \mathrm{~ms}$; IT, 1020 $\left.\mathrm{ms} ; \mathrm{FA}, 7^{\circ}\right]$ for coregistration purposes.

Preprocessing. Functional scans were corrected for slice scan time with cubic spline interpolation and for $3 \mathrm{D}$ head motion by aligning them to the first volume of their respective run with rigid body transformation (trilinear interpolation for parameter estimation and sinc interpolation for resampling). Linear trends and low-frequency drift were removed from the data with a temporal high-pass filter of 2 cycles/scan. We discarded the first four volumes of each functional run to remove T1saturated images from the time series data. We then used the first of the removed volumes as a reference for coregistration of all the functional data from the same run. Functional data were then spatially smoothed with a Gaussian kernel (of $4.5 \mathrm{~mm}$ FWHM) and spatially normalized across participants by transforming each dataset to standard Talairach space (Talairach and Tournoux, 1988).

Data analysis. We used BrainVoyager QX 2.0 (Brain Innovation) in combination with Matlab 7.11 (R2008b), IBM SPSS v.20, and Statistica 64 v.10 (Statsoft) for data analysis.

Behavioral performance. Accuracy data (in percentages of correct responses) were first entered in a two-way repeated-measures ANOVA with Session (practice, experimental) and Task (IIBT, IINT) as withinparticipants factors to test for possible differential effects of familiarity and scanning environment on task. Because we found a difference of accuracy between IIBT and IINT tasks during scanning, accuracy data for the experimental session were then entered in two separate two-way repeated-measures ANOVAs with Delay (No Delay, Delay) and Homology (No Homology, Partial Homology, Total Homology) as withinparticipants factors for each task. Accuracy data (in percentages of correct responses) for the targeted behavioral follow-up were entered in two separate two-way repeated-measures ANOVAs with Delay (No Delay, Delay) and Homology (No Homology, Partial Homology, Total Ho- 
mology) as within-participants factors for each session (i.e., standard or fMRI-like). Greenhouse-Geisser correction was applied whenever necessary. Interactions were disambiguated via planned comparisons, corrected with the Bonferroni-Holm method (Holm, 1979) unless otherwise stated.

Functional activation data. We entered brain activation data in a random effect (RFX) general linear model (GLM) analysis including the factors Task (IIBT, IINT), Delay (No Delay, Delay) and Homology (No Homology, Partial Homology, Total Homology). Each event type lasted $16 \mathrm{~ms}$ and was modeled by convolving the event timing with the dual $\gamma$ function. The resulting reference time courses were used to fit the $z$-transformed time course of each voxel. ROIs were selected by performing the IIBT > IINT contrast. Such contrast enabled the identification of brain regions showing a significantly higher response in the task needing BSRs than in the alternative task. Selecting ROIs based on this contrast does not allow any statements about whether any interactions between Delay and Homology occurred outside these regions. However, by not selecting the ROI based on Delay, Homology, or their putative interaction, we could perform unbiased testing of such effects in each ROI.

Statistical maps where corrected by applying a false discovery rate (FDR; Genovese et al., 2002) threshold of 0.05 (i.e., $q=0.05$, corresponding to $p<0.00001$ ), and by considering only clusters with $>8$ voxels as ROIs. $Z$-transformed $\beta$ estimates of the BOLD response for each condition were extracted separately for each ROI. The IIBT is a complex task and may concomitantly recruit different types of representations (e.g., primarily body structure representations but also auxiliary representations, such as mental images). Thus, to identify separate functional clusters, we performed a principal component analysis (PCA) on $\beta$ estimates from the ROIs that became significantly activated in the IIBT $>$ IINT contrast. We then followed that up with an analysis of individual component scores. For interpretation purposes, this provided complementary and converging evidence with the following analysis on parametric effects of homology.

To quantify the effects of homology as well as the effect of delay in all the ROIs, we then entered $\beta$ estimates into an overall repeated-measures ANOVA with ROI, Homology, and Delay as within-participant factors. Because a complex three-way interaction resulted $\left[F_{(16,192)}=3.230\right.$; mean squared error (MSE), $0.251 ; p<0.001$ ], we split the ANOVA into a series of separate Homology (No Homology, Partial Homology, Total Homology) by Delay (No delay, Delay) ANOVAs, one for each ROI, which we then followed up with linear contrasts and planned comparisons. For brain regions that showed a significant effect of Homology, further analyses were conducted to characterize the effect as either categorical (i.e., characterized as "all or none") or continuous (i.e., showing parametric modulation with homology degree) and establish which activations were more closely related with behavioral performance. A series of paired comparisons was then performed on the two ROIs showing parametric modulation with homology degree, to ascertain whether both of them showed significantly stronger overall signal than any other ROIs in the Delay condition. In each set of analyses, we used the BonferroniHolm procedure to correct for multiple comparisons. Finally, after Romo et al.'s proposal (2012), Pearson correlations between the homology effect in brain activations and in behavior were performed.

\section{Results}

\section{Behavioral performance}

In the practice session, stimuli were individually calibrated (see Materials and Methods) so that IIBT and IINT were of comparable difficulty as indicated by performance accuracy. Indeed, since we planned to subtract the pattern of brain activity during IINT from the pattern of brain activity during IIBT, having higher difficulty in IIBT (experimental task) than IINT (control task) might have confounded the resulting map of activations (scenario A). In that case, regions related to generic difficulty in addition to regions related to finger gnosis processing would have resulted in more activity in IIBT, and only finer analyses could have helped disambiguate the functional role of active regions (Corradi-Dell'Acqua et al., 2009). If instead the IINT (control task) was more difficult than IIBT (experimental task), then difficulty-related regions would result deactivated in the contrast, whereas the finger-related regions that are recruited by the IIBT and not (or at a lower extent) by the IINT may still result significantly more active (scenario B). Having an equal level of difficulty in IIBT and IINT would be ideal (scenario C). However, scenario B (i.e., IINT more difficult than IIBT) would be less problematic than scenario A (i.e., IIBT more difficult than IINT) for our hypothesis testing rationale.

In the practice session, outside the scanner room our participants were equally accurate in the IIBT (mean, 75\%; SE, 3\%) and the IINT (mean, 73\%; SE, 2\%) tasks $(t<1$; df $=13, p=0.592$ ).

However, we found that inside the scanner, accuracy for IIBT (mean, 84\%; SE, 3\%) significantly improved, whereas accuracy for IINT (mean, 69\%; SE, 2\%) remained constant. This was shown by a repeated-measure ANOVA with Session (practice, experimental) and Task (IIBT, IINT) as factors where the Session-Task two-way interaction was significant $\left(F_{(1,12)}=14.21\right.$; MSE, $41.7 ; p=0.003$; practice vs experiment: IIBT, $t_{(12)}=2.92$, $p=0.013$; IINT, $\left.t_{(12)}=1.45, p=0.173\right)$. This also meant that, in the experimental session, participants were more accurate in IIBT than in IINT $\left(t_{(12)}=5.40, p=0.0001\right.$; IIBT: mean, $84 \%$; SE, $3 \%$; IINT: mean, $69 \%$; SE, $2 \%$ ), presenting the situation described in scenario $\mathrm{B}$. We will now proceed with a more detailed description of our participants' performance in the IIBT and IINT during the experimental phase (i.e., inside the scanner).

In the IIBT, participants were more accurate with intermanual stimulation delay than with synchronous stimulation (Delay: mean, $88 \%$; SE, $3 \%$; No Delay: mean, $81 \%$; SE, $3 \% ; F_{(1,12)}=5.51$; MSE, $154 ; p=0.036$ ), and with stimulation of homologous fingers on the two hands than with partially homologous/nonhomologous configurations (No Homology: mean, 80\%; SE, 3\%; Partial Homology: mean, 84\%; SE, 3\%; Total Homology: 91\%; $\mathrm{SE}, 3 \% ; F_{(2,24)}=5.45$; MSE, $\left.160 ; p=0.011\right)$. However, the Homology effect was only present in the No Delay condition and was categorical (i.e., did not distinguish between the No Homology and Partial Homology condition but was sensitive to the difference between Total Homology and all the other conditions), as highlighted by the significant Delay-Homology interaction and paired $t$ tests $\left(F_{(2,24)}=6.45\right.$; MSE, $65 ; p=0.006$; No Homology and Partial Homology vs Total Homology; No Delay: $t_{(12)}=4.01$, $p=0.002$; Delay: $t<1$; Figure $3 A$ ).

In the IINT, performance was not modulated by Delay or Homology alone $\left(F<1\right.$ and $F_{(2,24)}=3.01$; MSE, 95; $p=0.068$, respectively). However, a small reverse categorical effect of Homology was present in the Delay condition, with participants showing higher accuracy in the No Homology and Partial Homology conditions than in the Total Homology condition, as highlighted by the significant Delay-Homology interaction and paired $t$ tests. Instead, no significant difference between levels of Homology was found in the No Delay condition $\left(F_{(2,24)}=3.62\right.$; MSE, 103; $p=0.042$; No Homology and Partial Homology vs Total Homology; Delay: $t_{(12)}=2.75, p=0.017$; No Delay: $t<1$; Figure 3B).

Note that whereas the IIBT results faithfully replicate Rusconi et al.'s (2009a) results, the IINT pattern of results (i.e., absence of the Homology effect in the No Delay condition and small but significant reverse Homology effect in the Delay condition) was unpredicted. It is possible that in the current fMRI study, the co-occurrence of major changes from the initial practice environment to the scanning environment-such as noise, posture, and temperature changes - might have selectively taxed the IINT when performed in the scanner. Indeed, whereas performance in 
A

IIBT

Delay by Homology

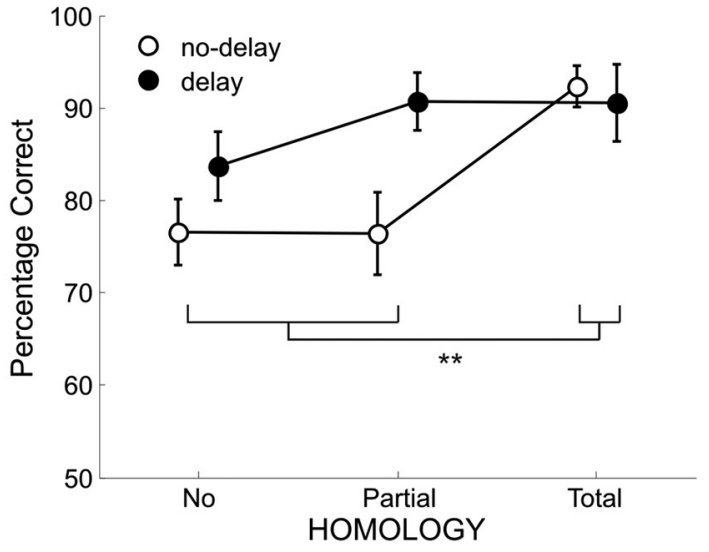

B
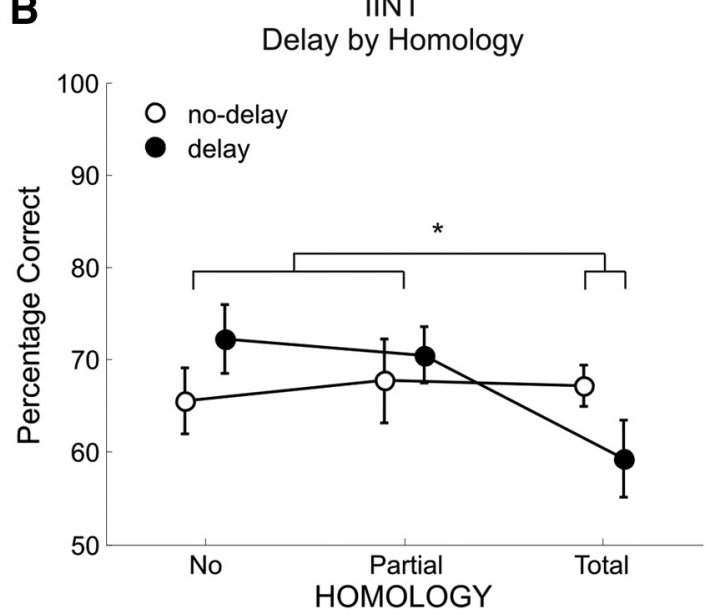

Figure 3. $A, \boldsymbol{B}$, Line plots show the percentage of accuracy in $(\boldsymbol{A})$ IIBT as a function of Delay and Homology; $(\boldsymbol{B})$ IINT as a function of Delay and Homology. Vertical bars represent the SEM and asterisks indicate significant differences between the means $\left({ }^{* *} p<0.005\right.$ and $\left.{ }^{*} p<0.05\right)$.

the IIBT appeared to benefit from the prolonged practice (overall accuracy increased from practice to the scanning session), performance in the IINT did not. To ascertain that the absence of a Homology effect in the fMRI environment, rather than some unaccounted sample characteristic or even statistical error, did play a role in the behavioral results of our fMRI study, we then tested an independent group of participants performing the IINT under standard (i.e., practice-like) and then fMRI-like environmental conditions. A significant Delay by Homology interaction was found in the practice-like setting $\left(F_{(2,18)}=4.31\right.$; MSE, 114; $p=0.03)$ but not in the fMRI-like setting $(p>0.14)$, where only the main effect of Delay reached significance $\left(F_{(1,9)}=6.41\right.$; MSE, $198 ; p=0.03$ ), indicating better accuracy for the Delay (mean, $84 \%$; SE, $3 \%$ ) compared with the No Delay condition (mean, $74 \%$; SE, 5\%). Planned $t$ tests revealed a regular Homology effect in the No Delay condition [No Homology (mean, 69\%; SE, 5\%) and Partial Homology (mean, 70\%; SE, 6\%) versus Total Homology (mean, $82 \%$; SE, $5 \%$ ): $t_{(9)}=2.27, p<0.05$ ] and no Homology effect in the Delay condition of the practice-like setting $(p>$ 0.62 ), similar to Rusconi et al. (2009a). In contrast, planned $t$ tests revealed no Homology effect in either timing condition for the fMRI-like setting ( $p s>0.73$ ). To summarize, with an independent sample of 10 participants performing the IINT both in standard and fMRI-like conditions, we could replicate the behavioral pattern reported by Rusconi et al. (2009a) for the Delay and the No Delay trials in the standard condition, and the current behavioral pattern for the No Delay trials in the fMRI-like condition. The smaller reverse Homology effect found in fMRI for the Delay condition was not replicated-which points to a possible type-I error in the original data.

BOLD response to explicit finger gnosis processing (IIBTspecific activation)

To identify the broad network of areas that are activated during an explicit finger gnosis task, we performed a $t$ contrast between activations in IIBT and IINT (RFX, GLM; FDR $q<0.05$; voxel size, $>8$ ). Figure 4 shows the statistical maps resulting from this contrast. All differentially active brain regions were located in the parietal and in the frontal cortices. We did not find any differential BOLD signals in the occipital or in the temporal cortices. In the parietal cortex, the left anteroventral inferior parietal lobule (a-vIPL), left anteromedial IPL (a-mIPL), and bilateral precu- neus (PCN) yielded a stronger BOLD signal in the IIBT than in the IINT; in the frontal cortex, the bilateral premotor cortex (PMC) and left inferior frontal gyrus (IFG) resulted in a stronger BOLD signal in the IIBT than in the IINT (Fig. 4A). Finally, the right superior temporal sulcus (STS) and left posterior cingulate cortex (PCC) resulted in a stronger BOLD signal in IINT than in IIBT (Fig. 4B). Table 1 shows Talairach coordinates (Talairach and Tournaux, 1988), cluster size, and nearest Brodmann area for this contrast.

To identify functional clusters that could reveal the substrates of processing subcomponents and/or the use of auxiliary networks (e.g., for visuospatial mental images) and individual differences in the relation between clusters, we performed a PCA with oblique rotation (oblimin, $\delta=0$ ) on $\beta$ estimates extracted from the $z$-transformed voxel time courses from the seven activated ROIs shown in Figure $4 A$. The Kaiser-Meyer-Olkin (KMO) measure, 0.773 , verified that sampling adequacy was good for the analysis (Kaiser, 1970; Hutcheson and Sofroniou, 1999; Field, 2009), and all KMO values for individual items were $\geq 0.599$, which is above the acceptable limit of 0.5 (Field, 2009). Bartlett's test of sphericity $\left(\chi_{(21)}^{2}=58.252, p<0.001\right)$ indicated that correlations between items were sufficiently large for PCA. Three identified components had eigenvalues $>0.6$ and in combination explained $90 \%$ of the variance. In Figure 5, we present factor loadings with values $>0.40$ after rotation. The ROIs that cluster on the same components suggest a topographical organization, with Component 1 representing bilateral frontal lobe regions, Component 2 representing bilateral dorsal parietal regions, and Component 3 representing left IPL regions (Fig. 5). Finally, as shown in Figure 5, we found that Component 2 and Component 3 correlated with Component 1 more strongly than they correlated with each other. This may suggest distinct functional contributions from left IPL and bilateral dorsal parietal regions, feeding into the frontal lobes. In relation to whether such contributions are both necessary to the task or rather fulfil a partly overlapping function (see Discussion), a closer look at individual component scores revealed that two different groups of participants could be identified in our sample based on the difference between scores on Component 2 and Component 3. In 6 of 13 participants, indeed, the difference was positive, indicating higher scores for the bilateral dorsal parietal ROIs than for the left IPL ROIs, and significantly different from zero (mean, 1.10; SE, 
$0.18 ; t_{(5)}=6.19 ; p=0.002$; corrected $\alpha$-level, 0.017). In the remaining 7 participants, the difference was negative, indicating higher scores for the left IPL ROIs than for the bilateral dorsal parietal ROIs, and significantly different from zero (mean, -0.94 ; SE, $0.25 ; t_{(6)}=3.83 ; p=$ 0.009 ; corrected $\alpha$-level, 0.017$)$. No difference was present between groups in their scores on Component 1 (Group 1: mean, 0.07; SE, 0.29; Group 2: mean, -0.06; SE, $1.25 ; t_{(11)}<1$; see Discussion).

\section{Modulation of BOLD response as a function of Homology and Delay in IIBT}

We then investigated whether hemodynamic response in the IIBT-related ROIs varied as a function of Homology and of the time occurring between the two stimulations (i.e., delay) in each ROI. More precisely, we assessed whether the BOLD response, as captured by $\beta$ estimates for $z$-transformed voxel time course, decreased as a function of Homology as this would indicate that only higher-level (as opposed to primary sensory) information about finger gnosis passed through that ROI during IIBT. This pattern was expected to appear exclusively in the No Delay condition, where participants could perform the IIBT for Total Homology trials without accessing a higher-level representation of body structure (i.e., without relying on finger gnosis). Information that is necessary to detect tactile stimulation on homologous fingers, indeed, is available to primary somatosensory cortex when bilateral stimulation occurs synchronously (Iwamura et al., 2001). Moreover, the presence of a parametric variation (i.e., discriminating between Partial and No Homology conditions) with Homology in the No Delay condition would indicate the most sensitive regions and core substrates of a BSR for the fingers. In other terms, when lower-level processing is sufficient to perform the task (i.e., No Delay, Total Homology), finger gnosis sites will be only slightly engaged; when lower-level processing can only partially contribute to the task (i.e., No Delay, Partial Homology), finger gnosis sites will play a more crucial role; when lower-level processing does not hold any key information (i.e., No Delay, No Homology), higher-level finger gnosis substrates will have to be relied upon. No low-level alternative is present in the Delay condition, because primary somatosensory codes will have decayed long before the chosen intermanual stimulation delay (Iwamura et al., 2001; Wühle et al., 2011), therefore

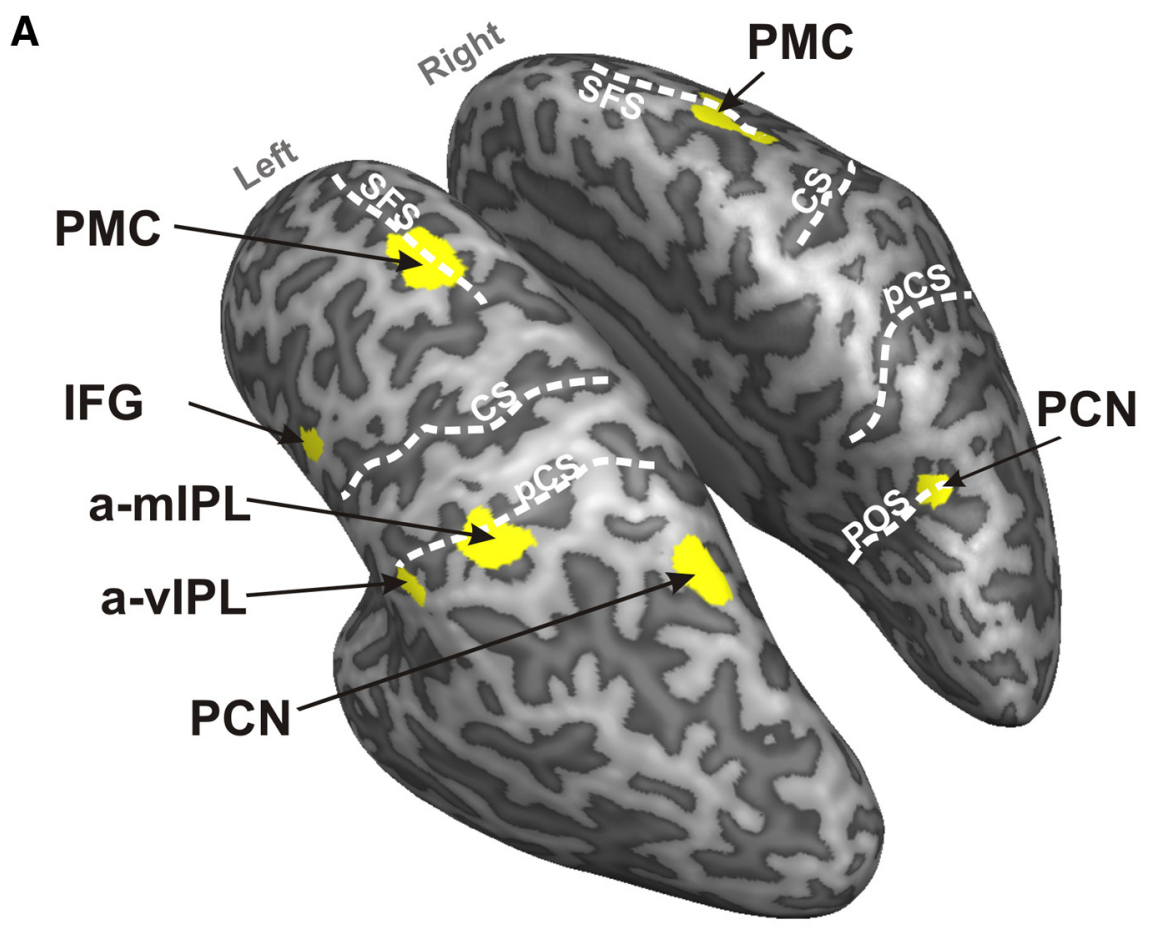

IIBT $>$ IINT $($ FDR $q<.05, N=13)$

B

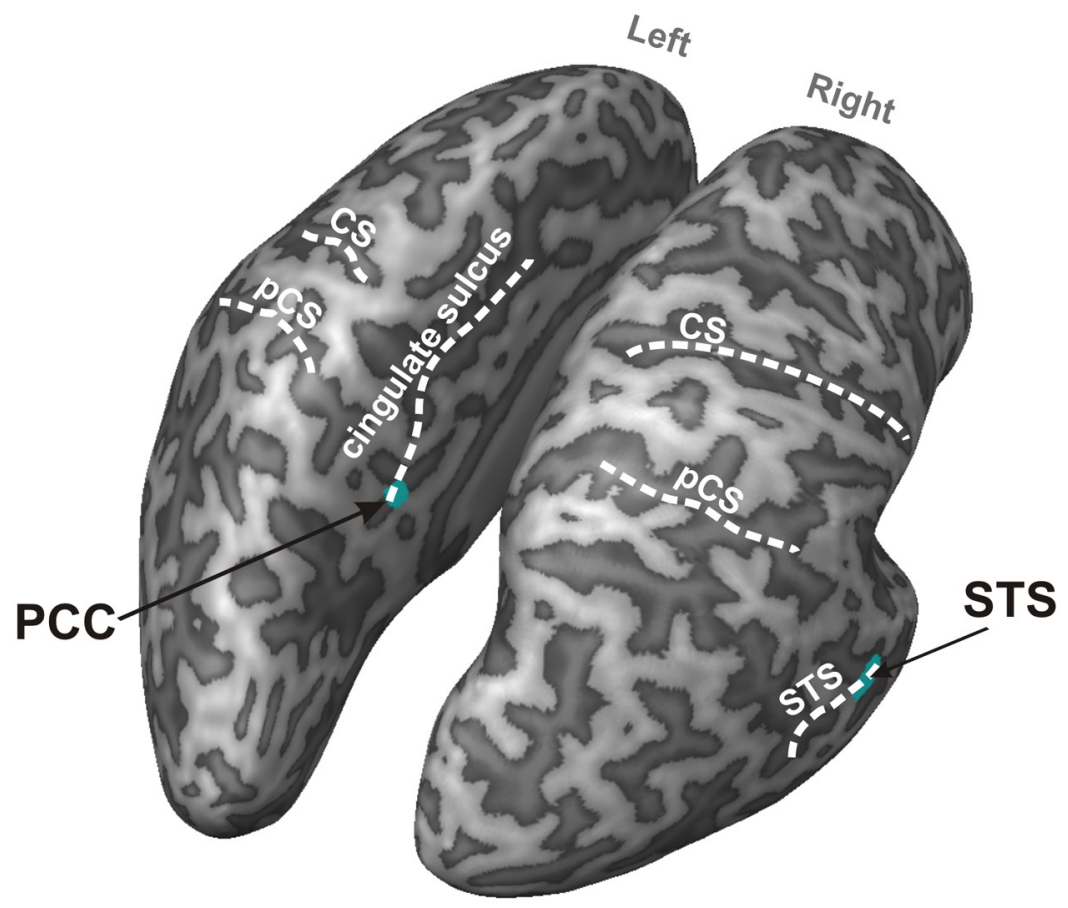

IINT $>$ IIBT (FDR q < .05, N = 13)

Figure 4. A, Statistical map showing brain regions with larger hemodynamic response for IIBT compared with IINT. Group functional data for the $t$ contrast corrected using FDR $q<0.05$ and cluster size $>8$ voxels are superimposed on the segmented and inflated left and right hemispheres of one participant. $\boldsymbol{B}$, Statistical map showing brain regions with smaller hemodynamic response for IIBT compared with IINT. Group functional data for the $t$ contrast corrected using FDR $q<0.05$ and cluster size $>8$ voxels are superimposed on the segmented and inflated left and right hemispheres of one participant (Table 1). 


\begin{tabular}{|c|c|c|c|c|c|c|c|}
\hline \multirow[b]{2}{*}{ ROls } & \multicolumn{3}{|c|}{ Mean $\pm S D$ center of mass } & \multirow{2}{*}{$\begin{array}{l}\text { Number } \\
\text { of voxels }\end{array}$} & \multirow{2}{*}{$\begin{array}{l}\text { Nearest } \\
\text { Brodmann } \\
\text { area }\end{array}$} & \multirow[b]{2}{*}{$t_{(12)}$} & \multirow[b]{2}{*}{$p$} \\
\hline & $X$ & $Y$ & $Z$ & & & & \\
\hline PMC left hemisphere & $-25.06 \pm 4.51$ & $-7.49 \pm 3.77$ & $54.19 \pm 3.54$ & 1982 & 6 & 11.006 & $<0.0001$ \\
\hline PMC right hemisphere & $24.38 \pm 2.77$ & $-7.37 \pm 4.33$ & $52.72 \pm 3.36$ & 1587 & 6 & 9.581 & $<0.0001$ \\
\hline IFG left hemisphere & $-49.93 \pm 4.15$ & $3.50 \pm 2.88$ & $29.21 \pm 2.85$ & 636 & 9 & 14.553 & $<0.0001$ \\
\hline a-vIPL left hemisphere & $-53.46 \pm 2.30$ & $-29.29 \pm 2.16$ & $33.59 \pm 2.84$ & 451 & 40 & 7.822 & $<0.0001$ \\
\hline a-mIPL left hemisphere & $-35.57 \pm 2.21$ & $-37.52 \pm 4.07$ & $39.80 \pm 3.40$ & 1061 & 40 & 6.943 & $<0.0001$ \\
\hline PCN left hemisphere & $-17.97 \pm 2.50$ & $-63.43 \pm 4.38$ & $48.35 \pm 2.98$ & 1026 & 7 & 7.173 & $<0.0001$ \\
\hline $\mathrm{PCN}$ right hemisphere & $17.69 \pm 3.47$ & $-61.38 \pm 2.50$ & $43.92 \pm 4.18$ & 1215 & 7 & 7.455 & $<0.0001$ \\
\hline STS right hemisphere & $42.94 \pm 2.36$ & $-28.98 \pm 1.44$ & $-0.24 \pm 2.10$ & 281 & 22 & -9.111 & $<0.0001$ \\
\hline PCC left hemisphere & $-4.18 \pm 1.78$ & $-43.95 \pm 1.54$ & $20.75 \pm 1.40$ & 127 & 30 & -8.144 & $<0.0001$ \\
\hline
\end{tabular}

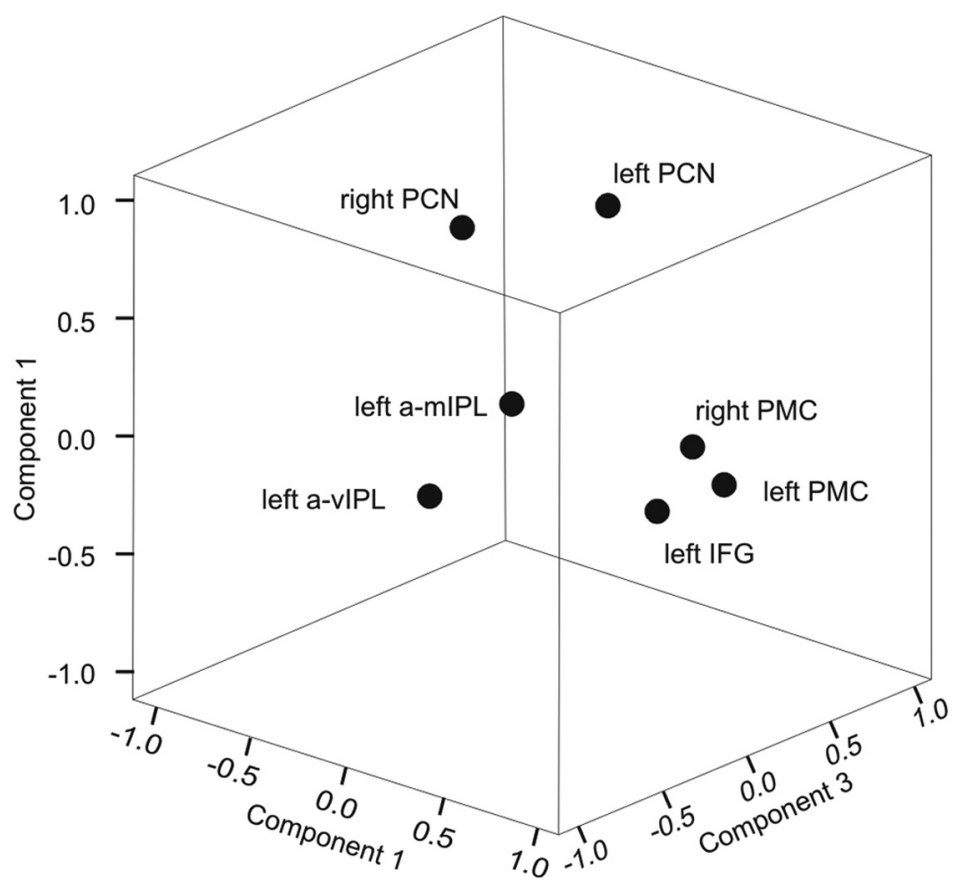

\begin{tabular}{|c|c|c|c|}
\hline \multicolumn{4}{|c|}{ Loadings (> .40) } \\
\hline \multirow[t]{2}{*}{ ROI } & \multicolumn{3}{|c|}{$\begin{array}{l}\text { Rotated Component Matrix } \\
\text { Component }\end{array}$} \\
\hline & 1 & 2 & 3 \\
\hline Left PMC & .965 & & \\
\hline Right PMC & .845 & & \\
\hline Left a-mIPL & & & 490 \\
\hline Left a-vIPL & & & .855 \\
\hline Left IFG & .839 & & \\
\hline Left PCN & & 913 & \\
\hline Right PCN & & .902 & \\
\hline
\end{tabular}

Extraction method: Principal Component Analysis Rotation Method: Oblimin (Kaiser Normalization) Rotation converged in 11 interactions

\begin{tabular}{|c|c|c|c|}
\hline \multicolumn{4}{|c|}{ Component Correlation Matrix } \\
\hline Component & 1 & 2 & 3 \\
\hline 1 & - & & \\
\hline 2 & .516 & - & \\
\hline 3 & .518 & .292 & - \\
\hline
\end{tabular}

Figure 5. ROls are shown in 3D space as function of the components identified with PCA. Three clusters are visible and characterized by higher scores along a specific component: Component 1 , frontal ROls; Component 2, dorsal parietal ROls; Component 3, left IPL ROIs. All loadings $>0.40$ are tabulated next to the plot, as well as correlations between components.

no modulation with Homology is expected in the Delay condition. In the Delay condition, core substrates of finger gnosis should be characterized by an overall stronger signal compared with other ROIs.

Figure 6 shows $\beta$ estimates as a function of Homology and Delay for all ROIs. Timing of stimulation affected the BOLD response in all of the ROIs. When pairs of tactile stimuli were delivered simultaneously to the two hands, the BOLD response was always higher in the IIBT-related ROIs, compared with when pairs of tactile stimuli were temporally offset between the two hands. A significant main effect of Delay was also found in the left PCC and in the right STS, indicating larger BOLD decrease for the No Delay condition (Table 3 , summary statistics for significant effects).

Also Homology modulated the BOLD response in all of the activated ROIs, with the BOLD response decreasing linearly as a function of Homology (Tables 4, summary statistics for the homology main effects, 5, summary statistics for post hoc linear contrasts). No main effect of Homology was found in the ROIs showing deactivation ( $p s>0.38$ ), which is consistent with their IINT-specific role rather than their IIBT-specific role.
Homology effects on BOLD response were modulated by Delay in all of the activated ROIs, as indicated by the significant Homology-Delay two-way interaction (Table 6, summary statistics), with a monotonic decrease of the BOLD response with increasing homology that was more pronounced in the No Delay condition (Table 7, post hoc linear contrasts on the HomologyDelay interaction). No interaction was found in the ROIs with negative BOLD response ( $p s>0.37)$.

Across brain regions where the Homology-Delay interaction was significant, we performed a series of Bonferroni-Holms-corrected $t$ tests between the No Homology and Partial Homology condition to identify the regions, if any, that may be parametrically related to Homology. These would indeed be the most likely candidate loci for the link between somatosensory processing to higher-level body representations (see Introduction and Discussion). As shown in Table 2, only two ROIs, the left a-mIPL and left PCN, showed a significant difference between No Homology and Partial Homology with a familywise significance threshold fixed at $\alpha_{\mathrm{FW}}=0.05$. In other words, the left a-mIPL and left PCN responded to Homology in a parametric way, whereas other areas responded to Homology only in a categorical way, which is similar to the effect we 
found in behavioral performance and may simply represent a task-specific difficulty effect.

Moreover, a series of paired-samples $t$ tests was performed for the Delay condition on the $\beta$ weights (averaged across all Homology levels) of the two ROIs with parametric response to Homology against all of the other ROIs (Table 8). It emerged that the BOLD signal was significantly stronger in the left a-mIPL than in any other region, including the left $\mathrm{PCN}$. The BOLD signal in the left PCN for the Delay condition was significantly higher than the right STS and left PCG but lower than in any other ROIs - and significantly so compared with left IFG and left PMC.

Finally, following Romo et al. (2012; de Lafuente and Romo, 2005), the correlation between brain activity and behavioral performance in tactile judgments should be very weak upstream, where the target stimulus attribute is encoded with more precision, and become significant in PMC where the final steps of decision making take place (Romo et al., 2012). If the same principle was applicable to higher-level representations, we would expect activation in left a-mIPL and PCN (i.e., the only ROIs whose activity varies parametrically with homology) to be more loosely related to behavioral performance than activation in PMCs.

We calculated Pearson's correlation coefficients between the homology effect in brain activation (difference between Total Homology and No Homology, in $\beta$ estimates for $z$-transformed voxel time course) for each of these four regions and the homology effect in behavioral performance (difference between Total Homology and No Homology, in percentages of correct responses). We then plotted these Pearson's coefficients against $t$ values of the difference in brain activation between No Homology and Partial Homology (Table 2), as an index of finger discrimination accuracy (i.e., with higher $t$ values indicating finer tuning). As shown in Figure 7, left PCN and a-mIPL were finely tuned on stimulation homology but their homology activation pattern was very loosely related with the homology effect in behavioral performance. On the other hand, left and right PMCs were very loosely tuned on stimulation homology but their homology activation pattern was more closely related to the homology effect in behavioral performance.

\section{Discussion}

Impairment of a BSR for the fingers was first reported as a special case of autotopagnosia (i.e., limited to one's own fingers) in the context of a posterior parietal syndrome of the dominant hemisphere (Gerstmann, 1924, 1940; Rusconi et al., 2010). However, double dissociations were later found between finger agnosia and autotopagnosia (Goldenberg, 2000), thus pointing to an independent neural substrate supporting a BSR for the fingers. Kinsbourne and Warrington (1962) devised a classical battery of tests for finger gnosis, with nonverbal tasks deemed as more appropriate than ver-
Table 2.t test for paired samples on No Homology and Partial Homology conditions $^{a}$

\begin{tabular}{llllll}
\hline ROI & Mean & SE & $t$ & $\mathrm{df}$ & $p$ \\
\hline PMC LH & 0.18 & 0.34 & 0.515 & 12 & 0.616 \\
PMC RH & 0.16 & 0.29 & 0.536 & 12 & 0.602 \\
IFG LH & 0.26 & 0.29 & 0.912 & 12 & 0.380 \\
a-vIPL LH & 0.42 & 0.27 & 1.56 & 12 & 0.144 \\
a-mIPL LH $^{b}$ & 0.59 & 0.28 & 3.32 & 12 & 0.006 \\
PCN LH $^{b}$ & 1.40 & 0.39 & 3.56 & 12 & 0.004 \\
PCN RH & 0.31 & 0.32 & 0.975 & 12 & 0.349 \\
\hline
\end{tabular}

${ }^{a}$ Corrected minimum $\alpha$ threshold, 0.007 .

${ }^{b}$ Results significant.

bal and symbolic tasks. Here we used a modified version of their in-between task, the IIBT, developed for use with healthy adults (Rusconi et al., 2009a). The IIBT is a direct test of finger gnosis that also enables a subtle test of implicit finger identity processing. By manipulating the identity of the stimulated fingers on the two hands, the homology effect (i.e., an advantage for the condition in which stimulation involves two homologous fingers on the two hands compared with any other combination) appears in behavioral performance when stimuli are delivered synchronously on the two hands (Rusconi et al., 2009a). Parametrically varying homology in IIBT enabled us to distinguish processes 
Table 3. Homology $\times$ Delay ANOVAs significant effects, Delay main effect ${ }^{a}$

\begin{tabular}{llllr}
\hline ROI & $F$ & MSE & df & $p$ \\
\hline PMC left hemisphere & 47.995 & 3.23 & 1,12 & $<0.001$ \\
PMC right hemisphere & 87.881 & 2.11 & 1,12 & $<0.001$ \\
IFG left hemisphere & 51.904 & 4.82 & 1,12 & $<0.001$ \\
a-vIPL left hemisphere & 18.142 & 5.90 & 1,12 & 0.001 \\
a-mIPL left hemisphere & 92.535 & 3.84 & 1,12 & $<0.001$ \\
PCN left hemisphere & 28.322 & 1.69 & 1,12 & $<0.001$ \\
PCN right hemisphere & 32.803 & 2.69 & 1,12 & $<0.001$ \\
PCC left hemisphere & 21.390 & 2.69 & 1,12 & 0.001 \\
STS right hemisphere & 40.692 & 1.08 & 1,12 & $<0.001$ \\
\hline
\end{tabular}

${ }^{a}$ Corrected minimum $\alpha$ threshold, 0.005 .

All results were significant.

Table 4. Homology $\times$ Delay ANOVAs significant effects, Homology main effect ${ }^{a}$

\begin{tabular}{lclll}
\hline ROI & \multicolumn{1}{l}{ If } & MSE & df & \multicolumn{1}{c}{$p$} \\
\hline PMC left hemisphere $^{b}$ & 10.988 & 0.70 & 2,24 & $<0.001$ \\
PMC right hemisphere $^{b}$ & 10.979 & 0.83 & 2,24 & $<0.001$ \\
IFG left hemisphere $^{b}$ & 18.234 & 0.55 & 2,24 & $<0.001$ \\
a-vIPL left hemisphere $^{b}$ & 7.678 & 0.50 & 2,24 & 0.003 \\
a-mIPL left hemisphere $^{b}$ & 18.107 & 0.42 & 2,24 & $<0.001$ \\
PCN left hemisphere $^{b}$ & 12.418 & 0.63 & 2,24 & $<0.001$ \\
PCN right hemisphere $^{b}$ & 8.651 & 0.99 & 2,24 & 0.001 \\
PCC left hemisphere & - & - & - & - \\
STS right hemisphere & - & - & - & - \\
\hline
\end{tabular}

${ }^{a}$ Corrected min $\alpha$ threshold, 0.005 .

${ }^{b}$ Results significant.

Table 5. Post hoc linear contrasts for Homology ${ }^{a}$

\begin{tabular}{lrll}
\hline ROI & \multicolumn{1}{l}{ df } & $p$ \\
\hline PMC left hemisphere & 12.234 & 1,12 & 0.004 \\
PMC right hemisphere & 13.515 & 1,12 & 0.003 \\
IFG left hemisphere & 19.748 & 1,12 & 0.001 \\
a-VIPL left hemisphere & 9.923 & 1,12 & 0.008 \\
a-mIPL left hemisphere & 23.935 & 1,12 & 0.001 \\
PCN left hemisphere & 18.170 & 1,12 & 0.001 \\
PCN right hemisphere & 11.159 & 1,12 & 0.006 \\
\hline
\end{tabular}

${ }^{a}$ Corrected minimum $\alpha$ threshold, 0.007 .

All results were significant.

Table 6. Homology $\times$ Delay ANOVAs effects, Homology-Delay interaction ${ }^{a}$

\begin{tabular}{lcllc}
\hline ROI & $F$ & MSE & df & $p$ \\
\hline PMC left hemisphere $^{b}$ & 9.621 & 0.53 & 2,24 & 0.001 \\
PMC right hemisphere $^{b}$ & 8.457 & 0.60 & 2,24 & 0.02 \\
IFG left hemisphere $^{b}$ & 13.070 & 0.46 & 2,24 & $<0.0001$ \\
a-vIPL left hemisphere $^{b}$ & 4.151 & 0.57 & 2,24 & 0.028 \\
a-mIPL left hemisphere $^{b}$ & 10.277 & 0.43 & 2,24 & 0.001 \\
PCN left hemisphere $^{b}$ & 11.414 & 0.54 & 2,24 & $<0.0001$ \\
PCN right hemisphere $^{b}$ & 7.065 & 0.84 & 2,24 & 0.004 \\
PCC left hemisphere $^{\text {STS right hemisphere }}$ & - & - & - & - \\
\hline
\end{tabular}

${ }^{a}$ Corrected minimum $\alpha$ threshold, 0.005 .

${ }^{b}$ Results significant.

Table 7. Post hoc linear contrasts, Homology $\times$ Delay $^{a}$

\begin{tabular}{lrll}
\hline ROI & \multicolumn{1}{l}{ df } & $p$ \\
\hline PMC left hemisphere & 8.758 & 1,12 & 0.012 \\
IFG left hemisphere & 12.958 & 1,12 & 0.004 \\
a-mIPL left hemisphere & 14.220 & 1,12 & 0.030 \\
PCN left hemisphere & 12.958 & 1,12 & 0.004 \\
PCN right hemisphere & 8.483 & 1,12 & 0.013 \\
\hline
\end{tabular}

${ }^{a}$ Corrected minimum $\alpha$ threshold, 0.01 .

All results were significant. specifically linked to finger gnosis from those linked simply to tactile perception and localization.

Even the simplest tactile frequency discrimination tasks recruit a frontoparietal network of areas involved in sensory processing, working memory, comparison, decision making, and response selection (Romo and de Lafuente, 2013). We identified a network supporting a BSR for the fingers by contrasting activations in a task of finger gnosis, the IIBT, and a matched control task, the IINT. The IINT was identical to the IIBT for stimulation energy, pattern, timing, response selection, and motor requirements. Whether the IINT should be interpreted as an indirect task of BSR (i.e., implying BSR processing although not in a conscious way; Rusconi et al., 2009a) rather than a pure sensorymotor control task is unclear. We would argue that this depends on whether the IINT does or does not show effects of homology. Because our IINT data are rather equivocal, the IINT cannot be considered a pure sensory-motor control task. Our fMRI results could thus reflect the cognitive processing using the BSR and/or the explicit conscious access to it-an ambiguity that is very common in many perceptual tasks. Even with these caveats, the IIBT > IINT contrast can be expected to highlight at least a broad network for explicit finger gnosis processing, including a core and task-relevant representation of BSRs for the fingers.

In a relatively small sample of participants, a frontoparietal network emerged from such contrast, comprising a dorsal and a medial region bilaterally (PMC and PCN), along with three leftlateralized regions more ventrally (a-mIPL, a-vIPL, and IFG). Because the control task was more difficult than the experimental task (notwithstanding our efforts to titrate performance and identical accuracy levels in IIBT and IINT recorded during the practice phase), it is unlikely that the IIBT-related network could be explained by higher attentional load. It is more likely that the resulting activations reflected engagement of IIBT-specific functional components. In agreement with previous imaging studies on BSRs (Felician et al., 2004; Corradi-Dell'Acqua et al., 2009), part of this network resides in the left posterior parietal lobe.

Only two regions were less active in IIBT than in IINT: left PCC and right STS. These are known to be involved in online spatial processing, often in association with the PCN region, and in multimodal integration of sensory signals (Cavanna and Trimble, 2006; Beauchamp et al., 2008). In particular, the right STS is maximally responsive to a combination of sensory, tactile, and visual attributes of an attended stimulus, but is also responsive to unimodal stimulation (Beauchamp et al., 2008) and its functional relation with frontoparietal circuits can be modulated by task domain (e.g., Köhler et al., 1998).

A data-driven functional description of the IIBT-active network was achieved via $\mathrm{PCA}$, revealing three main putative functional components with topographical organization: frontal, superior parietal, and left-lateralized inferior parietal regions. Both parietal components correlated highly with the frontal component but more weakly with each other. Half of our participants showed higher scores in relation with the superior parietal component, half with the inferior parietal component. These subgroups did not differ in their frontal component scores though. This pattern may reflect interindividual differences in the use of two alternative and somewhat complementary strategies to perform the IIBT. One builds on the primary role of the left hemisphere and of the a-mIPL in particular as could be expected from lesion and stimulation studies (Rusconi et al., 2010). The other builds on a frontoparietal system comprising medial parietal (PCN) and dorsal frontal (PMC) regions and uses visuospatial imagery-e.g., an explicit finger schema - to support performance in the IIBT (Cavanna and Trimble, 2006). A functional coupling 
Table 8. Paired-samples $t$ test on $\beta$ weights (averaged across Homology levels) for the Delay condition for left a-mIPL and left PCN (i.e. the ROIs showing parametric variation with Homology in the No Delay condition) versus all of the other ROls ${ }^{a}$

\begin{tabular}{|c|c|c|c|c|c|}
\hline ROI & Mean & SD & $t$ & df & $p$ \\
\hline a-mIPL left hemisphere vs PMC left hemisphere ${ }^{b}$ & 0.46 & 0.10 & 4.66 & 12 & 0.001 \\
\hline a-mIPL left hemisphere vs PMC right hemisphere ${ }^{b}$ & 0.49 & 0.07 & 6.82 & 12 & 0.000 \\
\hline a-mIPL left hemisphere vs IFG left hemisphere ${ }^{b}$ & 0.34 & 0.09 & 3.72 & 12 & 0.003 \\
\hline a-mIPL left hemisphere vs a-vIPL left hemisphere ${ }^{b}$ & 0.56 & 0.08 & 6.55 & 12 & 0.000 \\
\hline a-mIPL left hemisphere vs PCN left hemisphere ${ }^{b}$ & 0.73 & 0.09 & 7.88 & 12 & 0.000 \\
\hline a-mIPL left hemisphere vs PCN right hemisphere ${ }^{b}$ & 0.69 & 0.07 & 9.38 & 12 & 0.000 \\
\hline a-mIPL left hemisphere vs STS right hemisphere ${ }^{b}$ & 1.31 & 0.10 & 13.43 & 12 & 0.000 \\
\hline a-mIPL left hemisphere vs PCG left hemisphere ${ }^{b}$ & 1.50 & 0.09 & 15.91 & 12 & 0.000 \\
\hline PCN left hemisphere vs PMC left hemisphere ${ }^{b}$ & -0.26 & 0.09 & -3.07 & 12 & 0.010 \\
\hline PCN left hemisphere vs PMC right hemisphere & -0.24 & 0.09 & -2.78 & 12 & 0.017 \\
\hline PCN left hemisphere vs IFG left hemisphere ${ }^{b}$ & -0.39 & 0.11 & -3.67 & 12 & 0.003 \\
\hline PCN left hemisphere vs a-vIPL left hemisphere & -0.17 & 0.14 & -1.22 & 12 & 0.247 \\
\hline PCN left hemisphere vs PCN right hemisphere & -0.04 & 0.06 & -0.593 & 12 & 0.564 \\
\hline PCN left hemisphere vs STS right hemisphere ${ }^{b}$ & 0.58 & 0.11 & 5.36 & 12 & 0.000 \\
\hline PCN left hemisphere vs PCG left hemisphere ${ }^{b}$ & 0.77 & 0.11 & 7.06 & 12 & 0.000 \\
\hline
\end{tabular}

${ }^{a}$ Corrected minimum $\alpha$ threshold, 0.003 .

${ }^{b}$ Results significant.

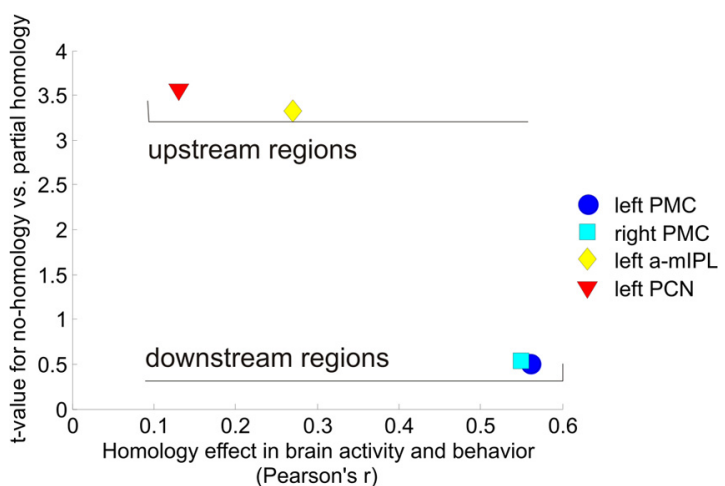

Figure 7. Scatterplot showing the relation between brain-behavior correlations for the Homology effect (calculated as a difference, in either percentage of correct responses or $\beta$ estimates, between Total Homology and No Homology conditions), and sensitivity to finger homology as exemplified by the $t$ value of the difference between Total Homology and Partial Homology (Table 2). As could be expected following Romo et al.'s (2012) reasoning, regions with higher sensitivity to stimulus characteristics (in this case, finger homology) are not as closely related to eventual behavioral performance as more downstream regions. "Upstream" and "downstream" labels are here used to indicate the relative position of associative brain substrates in the processing hierarchy, according to their functional role. Upstream regions extract task-relevant stimulus characteristics, whereas downstream regions translate received information into decision making.

between left and right hemispheres may be crucial in attuning the system to finger gnosis and/or amplifying and interfacing its core representation with a visuospatial finger schema, when finger gnosis is task-relevant and/or explicitly processed. This dual-route hypothesis found support in the following analyses.

We performed finer intra-ROI analyses based on the homology manipulation. We found a homology effect, consistent with the use of a lower-level shortcut for total homology trials, in the No Delay condition of the IIBT for behavioral performance (Rusconi et al., 2009a). No behavioral homology effect was instead found in the No Delay condition of the IINT, indicating that activation did not reach finger gnosis substrates, likely due to lack of resources (note that the BSR for the fingers was taskirrelevant in the IINT), or that it reached those substrates too slowly and/or did not trespass a critical threshold to influence behavioral performance. For completeness, we also report a small reverse homology effect in the Delay condition during IINT, with less accuracy in Total Homology trials than in Partial and No
Homology trials (but this was not replicated in a follow-up behavioral experiment with an independent sample of participants, and did not involve the crucial No Delay condition; see Results).

The signature homology effect in the No Delay condition could be traced in all IIBT-related regions. However, it was also possible to identify a more restricted set of ROIs whose response varied parametrically with homology levels. Because ROIs were identified by subtracting the more difficult from the less difficult task, the BOLD Homology effect does not represent generic difficulty. It could be a task complexity effect because homology is related to depth of processing. Differential processing is expected to occur in an all-or-none fashion, and the BOLD response to complexity should only discriminate between No Homology and Partial Homology versus Total Homology conditions. Sensitivity to parametric variations in homology may instead signal tuning to this high-level stimulus characteristic. Only two regions in the left parietal lobe showed that property: PCN and a-mIPL. This parametric variation indicates optimal tuning on finger identity, or the ability to discriminate among 0,1 , and 2 homologous fingers, as opposed to a categorical all-or-none classification in all of the other task-related regions. This is the type of tuning that we would expect to be associated with upstream finger gnosis. Also, if parametric responsiveness to homology was a valid indicator of upstream processing, the BOLD homology effect in the upstream regions should be more loosely connected than the BOLD homology effect in prototypical downstream areas (e.g., bilateral PMCs) with the behavioral homology effect (Romo et al., 2012). This was indeed the case (Fig. 7). Finally, left a-mIPL stood out as the most likely substrate for the retention of task-relevant information regarding finger gnosis because it was significantly more engaged than the left PCN in the Delay condition (Table 8).

Previous neuroimaging and lesion studies pointed to the left parietal lobe as "the" substrate for explicit body structure representation and bilateral finger gnosis (Mayer et al., 1999; Rusconi et al., 2005; Corradi-Dell'Acqua et al., 2008). A deficit, such as finger agnosia, can generally arise from lesions in the IPL territory (including lesions to supramarginal gyrus, angular gyrus, and/or posterior intraparietal connecting fibers; Rusconi et al., 2010). Pointing to the left a-mIPL as a primary substrate for finger gnosis is thus consistent with previous lesion and imaging studies, and suggests that the substrates of finger gnosis may not completely overlap with those of a BSR for the body (Corradi- 
Dell'Acqua et al., 2009). Its relatively anterior localization (i.e., closer to the somatosensory cortex, farther away from the visual cortex) may be rooted in a separate developmental trajectory of finger gnosis, which needs to be more finely tuned than other BSRs and concerns different exemplars of the same category rather than different categories of body parts. However, Rusconi and colleagues (2009b) have also suggested that finger gnosis may rest on intraparietal connectivity rather than on any isolated cortical region. Whether the PCN complex plays a crucial role in finger gnosis or simply contributes to performance in IIBT by providing an auxiliary visuospatial representation that can be interfaced, aligned with, and sometimes replace BSRs, as suggested here, will need to be further explored.

To conclude, our results show that both the left and the right hemisphere play a role in finger gnosis, thus confirming neuropsychological reports of finger agnosia after unilateral left and/or right parietal lesions (Gainotti et al., 1972). Left contributions may differ qualitatively from right contributions by providing an allocentric structural description of the fingers versus a representation of the fingers in egocentric space (Kinsbourne and Warrington, 1962). Within the left parietal lobe, we suggest that the interconnection between a-mIPL and PCN provides the core substrate for finger gnosis in IIBT, with the a-mIPL providing the primary substrate of a task-relevant BSR for the fingers and the PCN interfacing it with a visuospatial finger schema. Noninvasive brain stimulation techniques (Sandrini et al., 2011) will allow for a test of the suggested functional dynamics.

\section{References}

Allison T, McCarthy G, Wood CC (1992) The relationship between human long-latency somatosensory evoked potentials recorded from the cortical surface and from the scalp. Electroencephalogr Clin Neurophysiol 84: 301-314. CrossRef Medline

Beauchamp MS, Yasar NE, Frye RE, Ro T (2008) Touch, sound and vision in human superior temporal sulcus. Neuroimage 41:1011-1020. CrossRef Medline

Brainard DH (1997) The psychophysics toolbox. Spat Vis 10:433-436. CrossRef Medline

Cavanna AE, TrimbleMR (2006) The precuneus: a review of its functional anatomy and behavioural correlates. Brain 129:564-583. CrossRef Medline

Corradi-Dell'Acqua C, Hesse MD, Rumiati RI, Fink GR (2008) Where is a nose with respect to a foot? The left posterior parietal cortex processes spatial relationships among body parts. Cereb Cortex 18:2879-2890. CrossRef Medline

Corradi-Dell'Acqua C, Tomasino B, Fink GR (2009) What is the position of an arm relative to the body? Neural correlates of body schema and body structural description. J Neurosci 29:4162-4171. CrossRef Medline

de Lafuente V, Romo R (2005) Neuronal correlates of subjective sensory experience. Nat Neurosci 8:1698-1703. CrossRef Medline

De Renzi E, Scotti G (1970) Autotopagnosia: fiction or reality? Report of a case. Arch Neurol 23:221-227. CrossRef Medline

Felician $\mathrm{O}$, Romaiguère $\mathrm{P}$, Anton JL, Nazarian B, Roth M, Poncet M, Roll JP (2004) The role of human left superior parietal lobule in body part localization. Ann Neurol 55:749-751. CrossRef Medline

Field A (2009) Discovering statistics using SPSS, Ed 2. London: Sage.

Gainotti G, Cianchetti C, Tiacci C (1972) The influence of the hemispheric side of lesion on non verbal tasks of finger localization. Cortex 8:364-377. CrossRef Medline

García-Pérez MA (1998) Forced-choice staircases with fixed step sizes: asymptotic and small-sample properties. Vision Res 38:1861-1881. CrossRef Medline

Genovese CR, Lazar NA, Nichols T (2002) Thresholding of statistical maps in functional neuroimaging using the false discovery rate. Neuroimage 15:870-878. CrossRef Medline

Gerstmann J (1924) Eine umschriebene Störung der Orientierung am eigenen Körper. Wien Klin Wochenschr 1010-1012.

Gerstmann J (1940) Syndrome of finger agnosia, disorientation for right and left, agraphia and acalculia. Arch Neurol Psychiatry 398-407.
Goldenberg G (2000) Disorders of body perception. In: Patient-based approaches to cognitive neuroscience (Farah MJ, Feinberg TE, eds). Cambridge, MA: MIT.

Holm S (1979) A simple sequentially rejective multiple test procedure. Scand J Statistics 65-70.

Hutcheson G, Sofroniou N (1999) The multivariate social scientist. London: Sage.

Iwamura Y, Taoka M, Iriki A (2001) Bilateral activity and callosal connections in the somatosensory cortex. Neuroscientist 7:419-429. CrossRef Medline

Kaiser HF (1970) A second-generation little jiffy. Psychometrika 35:401415. CrossRef

Kinsbourne M, Warrington EK (1962) A study of finger agnosia. Brain 85: 47-66. CrossRef Medline

Köhler S, McIntosh AR, Moscovitch M, Winocur G (1998) Functional interactions between the medial temporal lobes and posterior neocortex related to episodic memory retrieval. Cereb Cortex 8:451-461. CrossRef Medline

Mauguière F, Merlet I, Forss N, Vanni S, Jousmäki V, Adeleine P, Hari R (1997) Activation of a distributed somatosensory cortical network in the human brain: a dipole modelling study of magnetic fields evoked by median nerve stimulation. Part II: effects of stimulus rate, attention and stimulus detection. Electroencephalogr Clin Neurophysiol 104:290-295. CrossRef Medline

Mayer E, Martory MD, Pegna AJ, Landis T, Delavelle J, Annoni JM (1999) A pure case of Gerstmann syndrome with a subangular lesion. Brain 122: 1107-1120. CrossRef Medline

Poeck K, Orgass B (1971) The concept of the body schema: a critical review and some experimental results. Cortex 7:254-277. CrossRef Medline

Romo R, de Lafuente V (2013) Conversion of sensory signals into perceptual decisions. Prog Neurobiol 103:41-75. CrossRef Medline

Romo R, Lemus L, de Lafuente V (2012) Sense, memory, and decisionmaking in the somatosensory cortical network. Curr Opin Neurobiol 22:914-919. CrossRef Medline

Rusconi E, Walsh V, Butterworth B (2005) Dexterity with numbers: rTMS over left angular gyrus disrupts finger gnosis and number processing. Neuropsychologia 43:1609-1624. CrossRef Medline

Rusconi E, Gonzaga M, Adriani M, Braun C, Haggard P (2009a) Know thyself: behavioral evidence for a structural representation of the human body. PLoS ONE 4:e5418. CrossRef Medline

Rusconi E, Pinel P, Eger E, LeBihan D, Thirion B, Dehaene S, Kleinschmidt A (2009b) A disconnection account of Gerstmann syndrome: functional neuroanatomy evidence. Ann Neurol 66:654-662. CrossRef Medline

Rusconi E, Pinel P, Dehaene S, Kleinschmidt A (2010) The enigma of Gerstmann's syndrome revisited: a telling tale of the vicissitudes of neuropsychology. Brain 133:320-332. CrossRef Medline

Sandrini M, Umiltà C, Rusconi E (2011) The use of transcranial magnetic stimulation in cognitive neuroscience: a new synthesis of methodological issues. Neurosci Biobehav Rev 35:516-536. CrossRef Medline

Schwarzbach J (2011) A simple framework (ASF) for behavioral and neuroimaging experiments based on the psychophysics toolbox for MATLAB. Behav Res Methods 43:1194-1201. CrossRef Medline

Schwoebel J, Coslett HB (2005) Evidence for multiple, distinct representations of the human body. J Cogn Neurosci 17:543-553. CrossRef Medline

Semenza C, Goodglass H (1985) Localization of body parts in brain injured subjects. Neuropsychologia 23:161-175. CrossRef Medline

Serino A, Haggard P (2010) Touch and the body. Neurosci Biobehav Rev 34:224-236. CrossRef Medline

Talairach J, Tournaux P (1988) Co-planar stereotaxic atlas of the human brain. New York: Thieme.

Tamè L, Farnè A, Pavani F (2011) Spatial coding of touch at the fingers: Insights from double simultaneous stimulation within and between hands. Neurosci Lett 487:78-82. CrossRef Medline

Tamè L, Braun C, Lingnau A, Schwarzbach J, Demarchi G, Li Hegner Y, Farnè A, Pavani F (2012) The contribution of primary and secondary somatosensory cortices to the representation of body parts and body sides: an AMRI adaptation study. J Cogn Neurosci 24:2306-2320. CrossRef Medline

Wühle A, Preissl H, Braun C (2011) Cortical processing of near-threshold tactile stimuli in a paired-stimulus paradigm-an MEG study. Eur J Neurosci 34:641-651. CrossRef Medline

Zeng H, Constable RT (2002) Image distortion correction in EPI: comparison of field mapping with point spread function mapping. Magn Reson Med 48:137-146. CrossRef Medline 